\title{
Trend of the research on rare earth elements in environmental science
}

\author{
Jonghoon Kang ${ }^{1}$ (D) $\cdot$ Albert M. Kang ${ }^{2}$
}

Received: 4 September 2019 / Accepted: 17 February 2020 / Published online: 22 February 2020

(C) Springer-Verlag GmbH Germany, part of Springer Nature 2020

\begin{abstract}
Rare earth elements (REEs) consist of 17 transition metals which are the 15 lanthanides and yttrium and scandium. These elements have great utility in the production of modern technology, especially electronics. However, these materials may pose a serious threat to the environment if handled or disposed of incorrectly; the effects of which are being studied by the field of environmental toxicology. A multitude of studies have indicated that rare earth elements have harmful impacts on biological life, making a reform to the disposal of rare earth elements increasingly pressing. Scientific interest in REEs is constantly rising due to the increased use of REEs due to their utility. In this paper, we display our meta-analysis of a scientific literature database, PubMed, to quantitatively map the temporal flux of research and interest pertaining to REEs, especially in the field of environmental science. Our findings may prove useful for planning research on REEs or predicting the future of REE usage.
\end{abstract}

Keywords Rare earth elements $\cdot$ Research trend $\cdot$ Scientometrics $\cdot$ PubMed $\cdot$ Regression analysis $\cdot$ Toxicity $\cdot$ Remediation

\section{Introduction}

The rare earth elements (REEs) are a series of seventeen transition metals: scandium, yttrium, and the lanthanoids, also known as the lanthanides, (lanthanum, cerium, praseodymium, neodymium, promethium, samarium, europium, gadolinium, terbium, dysprosium, holmium, erbium, thulium, ytterbium, and lutetium) (Connelly et al. 2005). REEs exhibit numerous peculiar physical and chemical properties, which ultimately arise from their distinctive electronic configurations (Barrett and Dhesi 2001). Because of those unique properties of REEs, they have been applied in various modern electronic industries, such as high-temperature superconductors, energysaving lamps, flat-screen monitors and televisions, rechargeable batteries, and very strong permanent magnets (Voncken 2016).

The growing usage of REEs in modern technologies accompanies unavoidable environmental concerns, as REEs

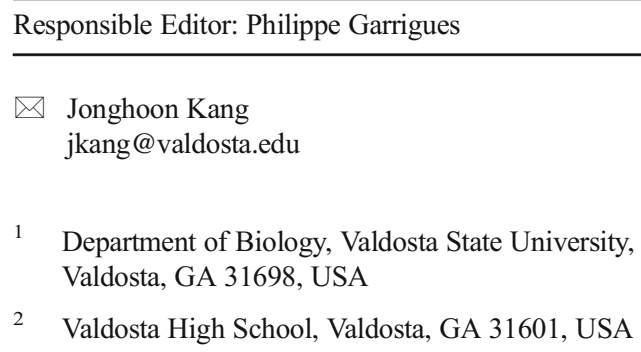

1 Department of Biology, Valdosta State University, Valdosta, GA 31698, USA

2 Valdosta High School, Valdosta, GA 31601, USA

have been found to exert toxic effects on various organisms (Turra 2018), while some REEs showed cytoprotective activity (Pałasz and Czekaj 2000). For example, many aquatic organisms were found vulnerable to toxic effects of REEs (RomeroFreire et al. 2019; Joonas et al. 2017). Some of the toxic effects of REEs were found to be mediated by gene expression changes (Dubé et al. 2019) or by directly binding to target proteins (Pałasz and Czekaj 2000). Numerous medical technologies have revealed that various neurophysiological processes including learning and memory were also affected by lanthanides (Pałasz et al. 2019; Jin et al. 2017). A recent review paper extensively summarized the ecological risks of REEs on plants, animals, and humans' health (Adeel et al. 2019).

The recurrent observations of biological toxicity of REEs have invoked another venue of research, which is the remediation of REEs from the environment. This includes phytoremediation (Wei et al. 2019; Jalali et al. 2019; Yuan et al. 2018) and nanoparticle-based remediation (Wang 2018; Su 2017), each of which is specialized for the removal of REEs in soil and water, respectively. In addition, bacteriabased remediation of REEs has been recently investigated (Cheng et al. 2018).

While research on REEs has been proliferating due to their applications to modern technologies and emerging concerns for their environmental contaminations, no quantitative analysis of the trend of the research is not available in literature. In 
our previous publications (Kang and Purnell 2011; Kang et al. 2015; Kang and Clifton 2018; Kang et al. 2019), we showed that the number of articles in a given academic/scientific field could be used as a measure of research activity of the field. PubMed search has been found as an effective way for the quantitative description of the research trend of various biomedical sciences such as biochemistry, biophysics, bioinformatics, food science, and epigenetics (Kang and Purnell 2011; Kang et al. 2015; Kang and Clifton 2018; Kang et al. 2019).

The rapid emergence of utilization of REEs in industry and the toxicity associated with them prompted us to characterize the development of research on REEs specifically in environmental science. We employed our PubMed-based approach to evaluate the trend of research on REEs by searching the database to quantitatively describe the development of the research. In this paper, we report our procedure and findings and share our perspective on the result with the readers of this journal.

\section{Materials and methods}

\section{PubMed search}

To examine the trend of research on REEs from a quantitative perspective, we searched the PubMed database, a search engine for biomedical publications archived in MEDLINE (https://www.ncbi.nlm.nih.gov/pubmed), using the search terms "rare earth elements" in June, 2019. From the search, the number of publications on REEs from 1947 to 2018 was obtained. However, these results may not all pertain to the same field of study, as multiple different fields mention the term "rare earth elements". Therefore, the publications obtained from this search were named "publications on general REE research". To retrieve papers of REE research specific to environmental science, which is the main objective of this study, a composite function was introduced in PubMed search with a search phrase, ("rare earth elements") AND (toxicology OR environment OR pollution). This search extracted papers in which the term "rare earth elements" was mentioned in the context of toxicology or environmental studies. The publications obtained from this search were named "publications on environmental REE research". The number of publications of each year was downloaded as a CSV (comma-separated values) file for further analysis of data.

\section{Regression analysis}

PubMed search results obtained as a CSV file were analyzed by regression analysis to obtain the equation of best fit using SigmaPlot (version 11; Systat Software, Inc., San Jose, CA). The search data were formatted on the Cartesian plane, with $x$ representing the year, the independent variable, and the publication number $(y)$ as the dependent variable: $y=f(x)$. To facilitate nonlinear regression, the year was subtracted by the number $\left(x_{0}\right)$ of 1 year prior to the year of the first publication: $y=g\left(x-x_{0}\right)$. The value of $x_{0}$ is 1946 for general rare earth element search and 1961 for that specifically related to environmental science.

\section{Results and discussion}

According to our search, the general REE research was found growing exponentially (Fig. 1) since its first publication in year 1947 (Meggers 1947). Regression analysis indicated that the data were best described by an exponential equation with a significant correlation $\left(R^{2} 0.9645\right)$ :

$y=a \times \exp \left[b \times\left(x-x_{0}\right)\right]$

where $y$ is the publication number at year $x$, and $x_{0}$ is the reference year. The fitting value and its standard error of each parameter obtained from nonlinear fitting are $0.0066 \pm 0.0027$ and $0.1403 \pm 0.006$ for $a$ and $b$, respectively. The reference year, $x_{0}$, is 1946. Plugging the values to the parameters and rearranging Eq. (1), the following equation was obtained for the general REE research trend:

$y=1.765 \times 10^{-121} \times \exp (0.1403 x)$

One should note that the coefficient in Eq. (2), $1.765 \times$ $10^{-121}$, is a very small number, which can be easily beyond the level of precision of the software in computational regression analysis. Thus, we modified the independent variable by subtracting the reference year from it in the regression analysis (see Materials and methods). Figure 1 shows the plot of Eq. (2). The plot clearly shows that the research on REEs has been exponentially growing. For example, the number of publications in the research at 2000 is 27 and it is 171 at 2018. In our

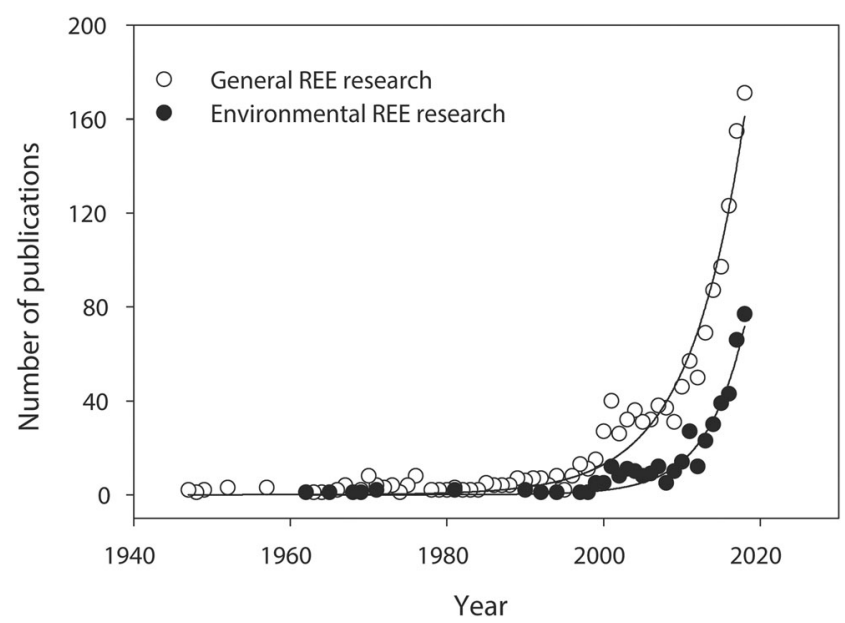

Fig. 1 Number of publications of REE research as a function of year 
previous research (Kang et al. 2015), we showed that regression analysis of PubMed search data allowed a reliable prediction of a short-term future (about 5 years). Based on Eq. (2), we can predict that the publication number in general REE research at 2023 in PubMed will be 325, double the amount in 2018.

We repeated the PubMed search to measure the research on REEs specifically in the context of environmental science, environmental REE research. Regression analysis indicated that the temporal dynamics of environmental REE research obtained from the PubMed search were also successfully described by Eq. (1) as its $R^{2}$ of the fitting is 0.9458 . The fitting values and standard errors of $a$ and $b$ are $0.0007 \pm 0.0006$ and $0.2024 \pm 0.0143$, respectively. The reference year, $x_{0}$, is 1961 . Equation (3) is the resulting equation for this case (Fig. 1):

$y=2.957 \times 10^{-176} \times \exp (0.2024 x)$

Equation (3) predicts that the number of publications in environmental REE research will be 197 at 2023, which is more than 2.5 times that of year 2018. The interest of environmental science in the research of REEs can be assessed by its proportion in the total REE research in terms of publication number (=number of publications in environmental REE research/number of publications in general REE research). It can be described by the ratio of Eq. (3) to Eq. (2), which produces Eq. (4):

$y=1.675 \times 10^{-53} \times \exp (0.0621 x)$

where $y$ is the proportion (\%) at year $x$ (Fig. 2). Based on the analysis, we can anticipate that the proportion will increase to $60 \%$ at 2023 from $45 \%$ at 2018, suggesting a growing importance of environmental science in the REE research.

While the growth of research on REEs was modeled as an exponential process in this paper, nothing can grow exponentially indefinitely. For example, research trend of biophysics

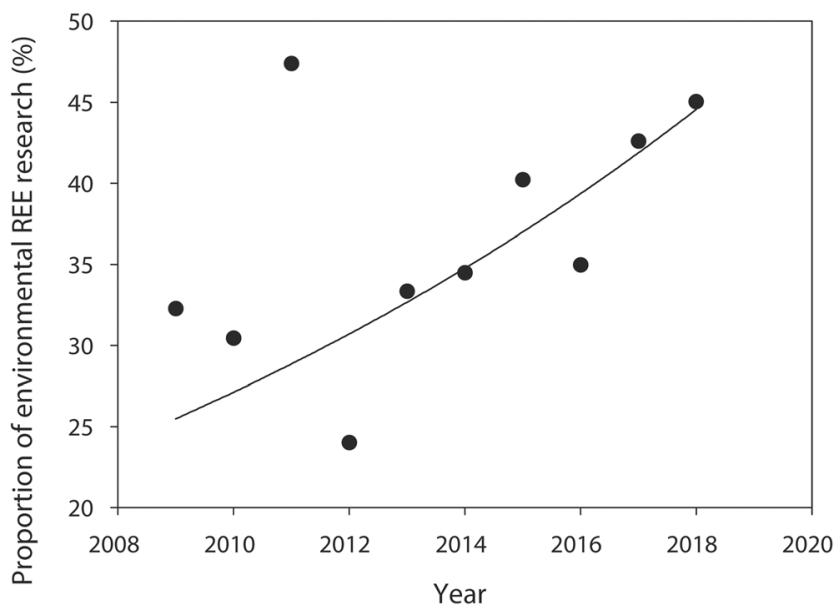

Fig. 2 Proportion of environmental science in REE research in the last 10 years was initially modeled as an exponential growth in our previous study (Kang and Purnell, 2011), but later it was found that a sigmoidal equation was more appropriate for the description of the research of biophysics (Kang et al. 2015). In our modeling of REE research trend, we found that fitting the data with sigmoidal equations did not produce reliable fitting parameter values in regression analysis of the data. This strongly suggests that the research activity on REEs both in general biomedical science and in environmental science is still in an early stage of a potential sigmoidal growth. While we expect that research on REEs will eventually behave in a sigmoidal mode, it is not feasible to predict when the research trend will switch to sigmoid from exponential behavior at present, which may be verified in future research.

In conclusion, analysis of PubMed search results in conjunction with nonlinear regression successfully identified that the temporal development of research on REEs could be described as an exponential process, and it is expected that REEs will remain one of the major topics in environmental science. Future directions in the REE research can be suggested based on our literature search. One obvious research area is the investigation of biological effects of REEs using biochemical, cell biological, and toxicological techniques (Pałasz and Czekaj 2000; Jin et al. 2017; Joonas et al. 2017; Turra 2018; Romero-Freire et al. 2019; Dubé et al. 2019; Pałasz et al. 2019). Another equally important field is the remediation of REEs in the environment (Su 2017; Yuan et al. 2018; Wang 2018; Cheng et al. 2018; Wei et al. 2019; Jalali et al. 2019). The findings introduced in this paper will be useful for planning research and education on REEs in environmental or related sciences.

Author contributions Jonghoon Kang contributed to the study conception and design. Material preparation, data collection, and data analysis were performed by Jonghoon Kang and Albert M. Kang. The first draft of the manuscript was written by Jonghoon Kang and all authors read and approved the final manuscript.

Funding information This work was supported by CoSM Research Scholar funding from Valdosta State University to Jonghoon Kang.

\section{References}

Adeel M, Lee JY, Zain M, Rizwan M, Nawab A, Ahmad MA, Shafiq M, Yi H, Jilani G, Javed R, Horton R, Rui Y, Tsang DCW, Xing B (2019) Cryptic footprints of rare earth elements on natural resources and living organisms. Environ Int 127:785-800. https://doi.org/10. 1016/j.envint.2019.03.022

Barrett SD, Dhesi SS (2001) The structure of rare-earth metal surfaces. Imperial College Press, London

Cheng Y, Zhang L, Bian X, Zuo H, Dong H (2018) Adsorption and mineralization of REE-lanthanum onto bacterial cell surface. Environ Sci Pollut Res Int 25:22334-22339. https://doi.org/10. 1007/s11356-017-9691-0 
Connelly NG, Damhus T, Hartshorn RM, Hutton AT (eds) (2005) Nomenclature of inorganic chemistry: IUPAC recommendations 2005. RSC Publishing, Norfolk

Dubé M, Auclair J, Hanana H, Turcotte P, Gagnon C, Gagné F (2019) Gene expression changes and toxicity of selected rare earth elements in rainbow trout juveniles. Comp Biochem Physiol C Toxicol Pharmacol 223:88-95. https://doi.org/10.1016/j.cbpc.2019.05.009

Jalali J, Gaudin P, Capiaux H, Ammar E, Lebeau T (2019) Fate and transport of metal trace elements from phosphogypsum piles in Tunisia and their impact on soil bacteria and wild plants. Ecotoxicol Environ Saf 174:12-25. https://doi.org/10.1016/j. ecoenv.2019.02.051

Jin C, Gao L, Li Y, Wu S, Lu X, Yang J, Cai Y (2017) Lanthanum damages learning and memory and suppresses astrocyte-neuron lactate shuttle in rat hippocampus. Exp Brain Res 235:3817-3832. https://doi.org/10.1007/s00221-017-5102-5

Joonas E, Aruoja V, Olli K, Syvertsen-Wiig G, Vija H, Kahru A (2017) Potency of (doped) rare earth oxide particles and their constituent metals to inhibit algal growth and induce direct toxic effects. Sci Total Environ 593-594:478-486. https://doi.org/10.1016/j.scitotenv. 2017.03.184

Kang J, Purnell CB (2011) Implications for undergraduate education of two interdisciplinary biological sciences: biochemistry and biophysics. CBE Life Sci Educ 10:111-112. https://doi.org/10.1187/cbe.1009-0124

Kang J, Park S, Venkat A, Gopinath A (2015) Quantitative analysis of the trends exhibited by the three interdisciplinary biological sciences: biophysics, bioinformatics, and systems biology. J Microbiol Biol Educ 16:198-202. https://doi.org/10.1128/jmbe.v16i2.949

Kang J, Clifton EC (2018) Quantitative analysis of food science trends. J Food Sci 83:2405-2406. https://doi.org/10.1111/1750-3841.13902

Kang J, Daines JR, Warren AN, Cowan ML (2019) Epigenetics for the 21st-century biology student. J Microbiol Biol Educ 20:20.3.56. https://doi.org/10.1128/jmbe.v20i3.1687

Meggers WF (1947) Electron configurations of "rare-earth" elements. Science 105:514-516. https://doi.org/10.1126/science.105.2733. 514
Pałasz A, Czekaj P (2000) Toxicological and cytophysiological aspects of lanthanides action. Acta Biochim Pol 47:1107-1114

Pałasz A, Segovia Y, Skowronek R, Worthington JJ (2019) Molecular neurochemistry of the lanthanides. Synapse. 73:e22119. https://doi. org/10.1002/syn.22119

Romero-Freire A, Joonas E, Muna M, Cossu-Leguille C, Vignati DAL, Giamberini L (2019) Assessment of the toxic effects of mixtures of three lanthanides (Ce, $\mathrm{Gd}, \mathrm{Lu})$ to aquatic biota. Sci Total Environ 661:276-284. https://doi.org/10.1016/j.scitotenv.2019.01.155

Su C (2017) Environmental implications and applications of engineered nanoscale magnetite and its hybrid nanocomposites: a review of recent literature. J Hazard Mater 322:48-84. https://doi.org/10. 1016/j.jhazmat.2016.06.060

Turra C (2018) Sustainability of rare earth elements chain: from production to food-a review. Int J Environ Health Res 28:23-42. https://doi. org/10.1080/09603123.2017.1415307

Voncken JHL (2016) The rare earth elements: an introduction. In the series: Springer briefs in earth sciences, Springer Nature, Dordrecht, The Netherlands

Wang J (2018) Adsorption of aqueous neodymium, europium, gadolinium, terbium, and yttrium ions onto nZVI-montmorillonite: kinetics, thermodynamic mechanism, and the influence of coexisting ions. Environ Sci Pollut Res Int 25:33521-33537. https://doi.org/10. 1007/s11356-018-3296-0

Wei Z, Hao Z, Li X, Guan Z, Cai Y, Liao X (2019) The effects of phytoremediation on soil bacterial communities in an abandoned mine site of rare earth elements. Sci Total Environ 670:950-960. https://doi.org/10.1016/j.scitotenv.2019.03.118

Yuan M, Liu C, Liu WS, Guo MN, Morel JL, Huot H, Yu HJ, Tang YT, Qiu RL (2018) Accumulation and fractionation of rare earth elements (REEs) in the naturally grown Phytolacca americana L. in southern China. Int J Phytoremediation 20:415-423. https://doi.org/ 10.1080/15226514.2017.1365336

Publisher's note Springer Nature remains neutral with regard to jurisdictional claims in published maps and institutional affiliations. 